\title{
BMJ Open Does adherence to a quality indicator regarding early weaning from invasive ventilation improve economic outcome? A single-centre retrospective study
}

\author{
Alexander Zuber, ${ }^{1,2}$ Oliver Kumpf, ${ }^{2}$ Claudia Spies (D) , ${ }^{2}$ Moritz Höft, ${ }^{2}$ Marc Deffland, ${ }^{2}$ \\ Robert Ahlborn, ${ }^{3}$ Jochen Kruppa, ${ }^{1}$ Roland Jochem, ${ }^{4}$ Felix Balzer (D) ${ }^{1,2}$
}

To cite: Zuber A, Kumpf 0 , Spies C, et al. Does adherence to a quality indicator regarding early weaning from invasive ventilation improve economic outcome? A single-centre retrospective study. BMJ Open 2022;12:e045327. doi:10.1136/ bmjopen-2020-045327

- Prepublication history and additional supplemental material for this paper are available online. To view these files, please visit the journal online (http://dx.doi.org/10.1136/ bmjopen-2020-045327).

Received 30 September 2020 Accepted 26 August 2021

Check for updates

(c) Author(s) (or their employer(s)) 2022. Re-use permitted under CC BY-NC. No commercial re-use. See rights and permissions. Published by BMJ.

${ }^{1}$ Institute of Medical Informatics, Charité Universitätsmedizin Berlin, Berlin, Germany ${ }^{2}$ Department of Anesthesiology and Operative Intensive Care Medicine, Charité Universitätsmedizin Berlin, Berlin, Germany

${ }^{3}$ IT Department, Charité Universitätsmedizin Berlin, Berlin, Germany

${ }^{4}$ Departments of Machine Tools and Factory Management, TU Berlin, Berlin, Germany

Correspondence to

Professor Felix Balzer;

felix.balzer@charite.de

\section{ABSTRACT}

Objectives To measure and assess the economic impact of adherence to a single quality indicator (QI) regarding weaning from invasive ventilation.

Design Retrospective observational single-centre study, based on electronic medical and administrative records. Setting Intensive care unit (ICU) of a German university hospital, reference centre for acute respiratory distress syndrome.

Participants Records of 3063 consecutive mechanically ventilated patients admitted to the ICU between 2012 and 2017 were extracted, of whom 583 were eligible adults for further analysis. Patients' weaning protocols were evaluated for daily adherence to quality standards until ICU discharge. Patients with $<65 \%$ compliance were assigned to the low adherence group (LAG), patients with $\geq 65 \%$ to the high adherence group (HAG).

Primary and secondary outcome measures Economic healthcare costs, clinical outcomes and patients' characteristics.

Results The LAG consisted of 378 patients with a median negative economic results of $-€ 3969$, HAG of 205 $(-€ 1030)$, respectively $(p<0.001)$. Median duration of ventilation was $476(248 ; 769)$ hours in the LAG and 389 $(247 ; 608)$ hours in the HAG $(p<0.001)$. Length of stay (LOS) in the LAG on ICU was $21(12 ; 35)$ days and 16 (11; 25) days in the HAG $(p<0.001)$. LOS in the hospital was 36 $(22 ; 61)$ days in the LAG, and within the HAG, respectively, $26(18 ; 48)$ days $(p=0.001)$.

Conclusions High adherence to this single $\mathrm{Ql}$ is associated with better clinical outcome and improved economic returns. Therefore, the results support the adherence to QI. However, the examined QI does not influence economic outcome as the decisive factor.

\section{INTRODUCTION}

In the last decades, the need for quality management $(\mathrm{QM})$ in the hospital has been growing. On one hand costs have been rising and on the other patients, health insurance and public pressure urge hospitals to improve outcome and services by cutting or tying reimbursement to valid quality indicators (QIs). ${ }^{1}$ This is why in the medium and long

\section{STRENGTHS AND LIMITATIONS OF THIS STUDY}

$\Rightarrow$ This is the first study evaluating whether a quality indicator on weaning has effects on the economic outcome parameters on a per case basis.

$\Rightarrow$ Results of the cost unit accounting practice is well established and is thus representative for a detailed examination of unit costs.

$\Rightarrow$ The test and validation sample was taken from a reference centre specialised on acute respiratory distress syndrome in adult patients with severe medical conditions.

$\Rightarrow$ Control for interactions with other quality indicators is necessary as the examined quality indicator is potentially connected with other ones.

$\Rightarrow$ The study results are based on German reimbursement system and might be typical for a tertiary university hospital rather than German hospitals in general

run quality-oriented reimbursement (pay for quality) might change the hospital landscape. ${ }^{2}$ Economics of health have been established widely in order to curb costs for the national healthcare system. Many countries introduced diagnosis-related groups (DRGs) in order to pay on averaged costs and on a generalised financial reimbursement per case (fixed prices). Reimbursement for inpatients is linked to DRG accounting and updated annually based on reported data from hospitals. The fee-for-service system induces hospitals to improve internal processes as reimbursement is predefined and to work goal-oriented towards therapeutic aims. ${ }^{3}$

In modern medicine, a considerable part of hospital costs arises from intensive care. The cost structure of a tertiary German hospital shows that ca. $20 \%$ of costs are generated in intensive care units (ICUs). ${ }^{4}$ Especially, mechanical ventilation is the main cost driver in ICUs. ${ }^{5}$ Approximately $6 \%$ of the patients in intensive care are affected by prolonged 
mechanical ventilation and weaning from mechanical ventilation represents an essential element in the treatment of critically ill patients as it can take up to $50 \%$ of the ventilation time. ${ }^{6}$ As a consequence, up to $37 \%$ of all ICU resources are allocated to these patients. ${ }^{7}$ This means that weaning patients from mechanical ventilation is not only essential for clinical outcomes like duration of ventilation or length of stay (LOS), ${ }^{89}$ but also a critical step from an economic perspective as costs can be reduced. Therefore, this process is a critical phase in intensive care. However, the ideal weaning process is still subject to debate. ${ }^{10}$ About $40 \%$ of patients receiving mechanical ventilation will experience a complicated weaning process. ${ }^{11}$ Patients categorised in prolonged weaning, failing at least three spontaneous breathing trials (SBTs) or receiving more than 7 days of weaning after the first SBTs, have an increased risk in developing hospital mortality, mainly through ventilator-associated pneumonia (VAP), ${ }^{6}$ but also through postintensive care syndrome (PICS) or chronic critical illness (CCI). ${ }^{12}$ Due to demographic changes and technological advances in intensive care, the number of older patients with complex diseases or comorbidities needing ventilation is increasing. ${ }^{13} 14$ This generates growing costs, as the cohort of patients requiring respiratory support accounts for a disproportionate percentage of the resources available in intensive care. ${ }^{15}$

With the purpose of managing quality throughout the difficult framework conditions of hospital care, a proactive and structured QM is essential. ${ }^{16}$ In general, QM focuses on securing and improving clinical services economically, performed by physicians or nurses according to the patient's needs. ${ }^{17}$ In Germany, in the context of European and national QM initiatives, consensus-based standardised QIs were developed for intensive care medicine since 2010 - third version in 2017-by the German interdisciplinary society for intensive and emergency care (DIVI) in order to simplify the measurement of relevant quality data, to record timely and to allow transparent comparisons of patient data. The according quantification of $\mathrm{QM}$ helps measuring effectiveness and efficiency of ICUs. ${ }^{18} 19$ QIs enable a descriptive picture of the actual condition and are an indispensable instrument for comparisons between different states of quality. ${ }^{18}$ Potentially, widely accepted QIs can progress hospital economics and support the reduction of the national budget for healthcare, even though a recent study has shown that costquality relationships are difficult to generate. ${ }^{20}$

QIs empower advances in intensive care medicine to be measured and evaluated on a regular basis. ${ }^{19}$ QIs can be defined as representative figures for quality of structure, processes or outcome within the medical care process. Thus, indicators are useful for measuring improvement in the context of QM and should be developed in line with evidence-based literature. ${ }^{21}$ Ideally, measures for QIs can be extracted from routine patient data to avoid excess documentation work. Therefore, patient data management systems (PDMS) are pivotal for measuring complex quality figures. ${ }^{18}$ The economic aspects for the whole hospital of the introduction of QIs are not well investigated. However, the is evidence that the application of QIs is a value-creating instrument. ${ }^{12}$

The objective of this study was, to determine the economic impact of adherence to a single QI evaluating the weaning process from invasive ventilation. We analysed this by comparing economic results per case and clinical outcome parameters like LOS between two groups of either high-quality or low-quality adherence. Additionally, we sought to determine factors that would influence a potential interaction between economic and outcome parameters.

\section{METHODS}

This original research is in accordance with the Consolidated Health Economic Evaluation Research Standards.

\section{Patient and public involvement}

Patients and the public were not directly involved in this observational study.

\section{Study centre}

We conducted this single study-centre in a university hospital (Charité-Universitätsmedizin Berlin). This observational analysis was performed at a 14-bed ICU (reference centre), specialised in treatment of acute respiratory distress syndrome in adult patients. All patients at our ICU were treated according to guidelines and internal standard operating procedures for clinical practice. ${ }^{22}$

\section{Study design}

This was a retrospective descriptive study, using data from multiple electronic databases used in routine patient care and for routine administrative purposes. All patients admitted to and discharged from the ICU between 1 January 2012 and 31 December 2017 who received invasive ventilation during their stay were eligible to be included in this study. Furthermore, duration of ventilation $<95$ hours, receiving no invasive ventilation, terminal status, incomplete patient record or missing readiness to be weaned were defined as exclusion criteria (see figure 1).

Confidentiality was guaranteed, no interventions were performed and only clinical routine data were collected. Data were retrieved from a PDMS called (Computer Organised Patient Report Assistant; COPRA System, Berlin, Germany). Data are recorded both automatically by patient monitors and manually by caregivers. The ICU staff validates all information manually. However, the design of the PDMS prevents manual alterations to the data, for example adding missing values after discharge from the ICU. PDMS data are also transferred to the clinical information and accounting system (SAP, Walldorf, Germany). Based on this administrative system, cost unit accounting is performed annually. In addition to basic demographic data, we assessed clinical and administrative parameters of in-patient cases (eg, LOS). Data were 


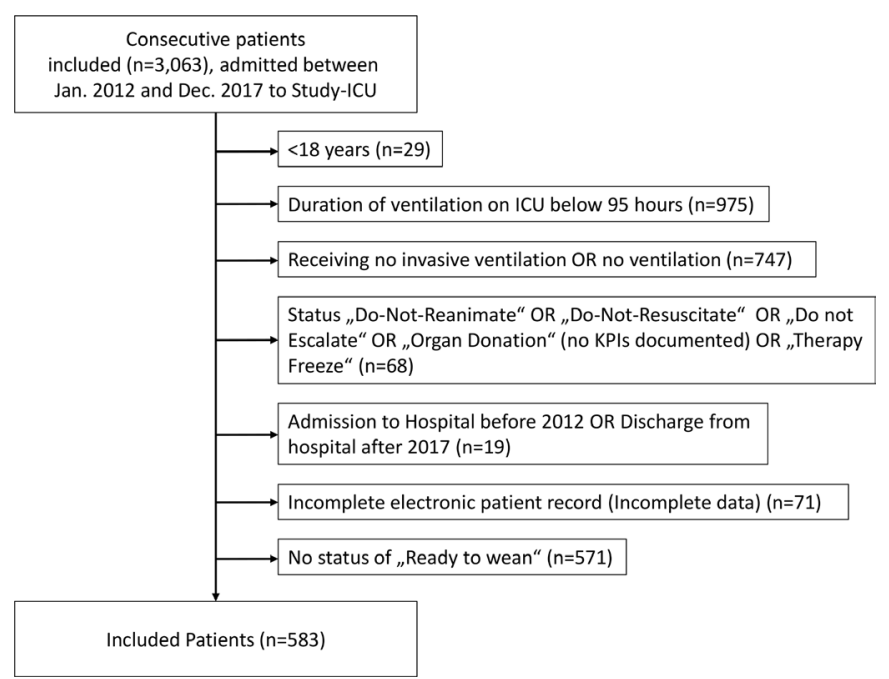

Figure 1 Patient inclusion and exclusion criteria. Flow chart of the process used in this study for patient record inclusion. Numbers listed are number of patients in each group. ICUs, intensive care units; KPIs, key performance indicators.

retrieved using a structured query. No patient identifiers were extracted in order to secure anonymity of patients' data. Data related to diagnoses were not retrieved from the administrative systems.

PDMS data of patients included in the study were transferred to the study database, where we also collected the administrative and cost accounting data, respectively. We contrasted patient, intensive care and economic parameters of the two adherence groups (see table 1). Then, we calculated the profits per case by subtracting costs of reimbursement per case. In order to generate an economic outcome per case for the dependent variable in multivariate linear regression. Besides administrative data, we used different scores for assessing the QI for eligibility. Selection criteria were: (1) no additional workload required for documentation, (2) the availability within the PDMS system, (3) standardised values for all patients and the existence of standard operating procedures for each indicator and (4) the relevance of the indicator for clinical decision making. ${ }^{19} 23$

\section{Procedures}

In this study, we used present key performance indicators (KPIs) in order to examine the adherence to the QI 'Early Weaning from Invasive Ventilation' until ICU discharge. ${ }^{8}$ A small set of evidence-based KPIs was established in 2009, providing indicators that were already available within the PDMS. The KPIs in intensive care medicine proved helpful for practical use and compliance with standard operating procedures. A description of the KPI is provided in online supplemental material. Within the weaning therapy, fast visual feedback for 'readiness to wean' and 'weaning protocol compliance' were implemented. If both KPIs were positive, the according result of the SBT was recorded. ${ }^{23}$ Once the patient was assessed to be ready to wean since the primary disease showed clinical improvement, the standard weaning protocol activities were conducted on a daily basis according to standard operating procedures. Congruent with clinical guidelines in place, weaning protocols were adapted to evaluate the progress of respirator therapy. ${ }^{22}$ The subsequent result was recorded in the weaning protocol. For each patient, we monitored the daily weaning protocol compliance between readiness to wean and ICU-discharge in order to evaluate the percentage of adherence. Within the weaning process, the SBT represents the major diagnostic test to evaluate if the patient can be extubated successfully. ${ }^{10}$ The SBT is successful if the patient succeeded the trial and does not have to be re-intubated within 48 hours. ${ }^{24}$ This process is directly linked to a specific QI for weaning derived from the DIVI-QI. ${ }^{19}$ A definition of the indicator is presented in online supplemental material.

\section{Outcome parameters}

In this study, we investigated for economic results, clinical outcome parameters per case and the respective adherence to quality. Economic results were defined as the profit or loss per case, by subtracting all assigned costs from the reimbursement on a case level. Clinical outcomes as a representative for clinical effectiveness were measured in order to set economic outcomes in relation to the purpose of medicine. Adherence to quality was calculated on a per case level in order to categorise the patients into groups.

We used the adherence level of the examined QI in order to create two quality groups. We calculated the final quality level by averaging the daily indicator results for the duration with equal weights per day. In order to set the optimal cut-off point for dichotomously distinguishing between high-adherence and low-adherence of weaning quality, we combined recommendations from literature with our institutional standards. A cut-off value of $70 \%$ deemed as a suitable fulfillment-threshold for QIs. ${ }^{25}$ However, due to partially high workload under certain circumstances in intensive care, we decided to lower the cut-off for 5\% tolerance in order to account for missing values in documentation. Therefore, we inserted a cut-off for weaning protocol compliance at $65 \%$ adherence. The low adherence group (LAG) was defined as adherence to QI of less than $65 \%$. The high adherence group (HAG) was defined as adherence to QI of equal or more than $65 \%$. Once this threshold was reached, the QI was characterised as high adherence.

\section{Statistical analyses}

Descriptive analyses and statistical testing were performed using SPSS, V.14.0 (SPSS) for Windows. Results are expressed as median (IQR) or frequency (\%). We controlled data for risk and severity by exclusion as patients and therapies in intensive care are heterogenic, as studies have shown. ${ }^{18}$ Differences between the adherence groups in terms of outcome parameters were tested using the univariate unpaired t-test and $\chi^{2}$ statistics for independent variables as appropriate with a $\mathrm{p}<0.05$ regarded as significant. 
Table 1 Patient demographics and outcome parameters in comparison between Ql adherence groups

\begin{tabular}{|c|c|c|c|c|}
\hline & All patients & $\begin{array}{l}\text { LAG } \\
\text { QI }<65 \%\end{array}$ & $\begin{array}{l}\text { HAG } \\
\text { QI } \geq 65 \%\end{array}$ & \\
\hline & $\mathrm{n}=583$ & $\mathrm{n}=\mathbf{3 7 8}$ & $\mathrm{n}=205$ & $P$ value \\
\hline \multicolumn{5}{|l|}{ Demographics } \\
\hline Age (years) & $57(40 ; 70)$ & $57(40 ; 70)$ & $55(42 ; 69)$ & 0.770 \\
\hline Gender (male) & $377(64.7 \%)$ & $233(61.6 \%)$ & $144(70.2 \%)$ & 0.038 \\
\hline \multicolumn{5}{|l|}{ ICU score on admission } \\
\hline APACHE II & $21(14 ; 27)$ & $21(15 ; 27)$ & $21(14 ; 27)$ & 0.986 \\
\hline SAPS II & $47(34 ; 61)$ & $47(35 ; 60)$ & $47(34 ; 62)$ & 0.860 \\
\hline SOFA & $9(7 ; 12)$ & $9(7 ; 12)$ & $9(7 ; 13)$ & 0.526 \\
\hline Average SOFA & $8.2(6.6 ; 10.3)$ & $8(6.5 ; 10.1)$ & $8.4(6.8 ; 10.7)$ & 0.140 \\
\hline Type of admission to Study-ICU & & & & 0.651 \\
\hline Medical & $290(49.7 \%)$ & $190(50.3 \%)$ & $100(48.8 \%)$ & \\
\hline Emergency surgery & $232(39.8 \%)$ & $146(38.6 \%)$ & $86(41.9 \%)$ & \\
\hline Elective surgery & $61(10.5 \%)$ & $42(11.1 \%)$ & 19 (9.3\%) & \\
\hline \multicolumn{5}{|l|}{ Outcome parameter } \\
\hline Duration of Ventilation Study-ICU (hour) & $431(250 ; 709)$ & $476(248 ; 769)$ & $389(247 ; 608)$ & $<0.001$ \\
\hline $\begin{array}{l}\text { Total duration of ventilation hospital } \\
\text { (hour) }\end{array}$ & $578(338 ; 924)$ & $597(310 ; 992)$ & $535(361 ; 821)$ & 0.017 \\
\hline No spontaneous breathing trials & $1(0 ; 2)$ & $1(0 ; 2)$ & $1(0 ; 2)$ & 0.456 \\
\hline No reintubation & $0(0 ; 1)$ & $0(0 ; 1)$ & $0(0 ; 1)$ & 0.531 \\
\hline Type of discharge of study-ICU & & & & $<0.001$ \\
\hline ICU & $161(27.6 \%)$ & $100(26.5 \%)$ & $61(29.8 \%)$ & \\
\hline Intermediate/ward & $260(44.6 \%)$ & $172(45.5 \%)$ & $88(42.9 \%)$ & \\
\hline Rehabilitation & $110(18.9 \%)$ & $56(14.8 \%)$ & $54(26.3 \%)$ & \\
\hline ICU mortality & $52(8.9 \%)$ & $50(13.2 \%)$ & $2(1.0 \%)$ & \\
\hline LOS Study-ICU (days) & $19(11 ; 32)$ & 21 12;35) & $16(11 ; 25)$ & $<0.001$ \\
\hline LOS hospital (days) & $33(20 ; 54)$ & $36(22 ; 61)$ & $26(18 ; 48)$ & 0.001 \\
\hline Profit $(€)$ & $-2999(-15946 ; 7730)$ & $-3696(-21170 ; 6828)$ & $-1030(-11$ 134; 9449) & $<0.001$ \\
\hline
\end{tabular}

Discrete variables are presented as a total number of encounters and were analysed with $\chi^{2}$ test for non-parametric samples.

APACHE II, Acute Physiology and Chronic Health Evaluation II; HAG, high adherence group; ICU, Intensive care unit; LAG, low adherence group; LOS, Length of stay; QI, quality indicator; SAPS, Simplified Acute Physiology Score; SOFA, Sequential Organ Failure Assessment.

In order to investigate the influencing factors in more detail, parameters that were found to be statistically significant on univariate analysis or out of discussion among the experts underwent stepwise multivariate analyses. We used multiple linear regression analyses to model the relationship between the independent variables and the outcome of profitability. Regression coefficients (95\% $\mathrm{CI}$ ) and the corresponding $\mathrm{p}$ values were calculated for each factor. Testing the dataset for outliers was performed using the cook distance test, based on the model. The test did not indicate the need to dismiss cases from the sample. Due to an exploratory character of the research, no adjustments for multiple testing were made.

\section{RESULTS}

All patients with complete electronic patient records $(\mathrm{n}=3063$ patients $)$ were screened for eligibility. After selection regarding inclusion and exclusion criteria, 583 patients were included in the final analysis (figure 1). Of these patients, 378 showed low-adherence if the indicator was below $65 \%$ and 205 showed high adherence. The median age of admitted patients was $57(40 ; 70)$ years; $64.7 \%$ of patients were male. There were significantly $(\mathrm{p}=0.038)$ more male patients within the HAG $(70.2 \%)$ than in the LAG $(61.6 \%)$. As reflected by a median Acute Physiology and Chronic Health Evaluation II (APACHE II) admission score of 21 (14; 27), a Simplified Acute Physiology Score II (SAPS II) admission score of 47 (34; 61) and a Sequential Organ Failure Assessment (SOFA) admission score of $9(7 ; 12)$, the study population was characterised by severe medical conditions. Patient 
demographics are displayed in table 1 . Along the line, at discharge patients generated an average daily SOFA score of 8.2 (6.6.; 10.3) indicating resource-intensive monitoring and treatment of the patient.

In order to account for the remaining clinical patient outcomes after grouping, we analysed the ventilation parameters. Overall in the median, patients were ventilated for 431 (250; 709) hours on the ICU and 578 (338; 924) throughout their hospital stay. Following the division into two adherence groups, there was a significant reduction in duration of ventilation on ICU from 476 to 389 hours $(\mathrm{p}<0.001)$. Overall in-hospital duration of ventilation was decreased from 597 to 535 hours $(\mathrm{p}=0.017)$. Concerning the number of SBTs and reintubations, there was no significant finding $(\mathrm{p}=0.456$ and $\mathrm{p}=0.531)$. In addition to the significant decrease in ventilation parameters seen between the differences in adherence, the LOS was decreased by 5 days from 21 to $16(\mathrm{p}<0.001)$ and overall in-hospital LOS decreased from 36 to 26 days per patient $(p=0.001)$ in the median, indicating strong arguments for QI adherence. With regard to economic outcome, the overall median economic results (loss) per case was $-€ 2999$. There was an increase in profitability from a median loss of $€ 3696-€ 1030(\mathrm{p}<0.001)$.

Considering the discharge of the patients, there was a highly significant difference $(p<0.001)$ between both groups. Most patients were discharged to intermediate care $(44.6 \%)$, other ICUs $(27.6 \%)$ or rehabilitation $(18.9 \%)$. Within the LAG, $50(13.2 \%)$ patients died on the ICU compared with $2(1.0 \%)$ in the HAG. This gives room to assume a certain impact of weaning quality on mortality. However, since we did not include diagnosis data, we cannot exclude an influence from this fact.

\section{Multiple linear regression}

The results of the multivariate linear regression analysis of the complete study population of 583 patients are given in table 2 . The parameters were not adjusted for severity

Table 2 Multiple linear regression analysis of factors affecting the profit of 583 intensive care patients who underwent the weaning process

\begin{tabular}{llrr}
\hline Variable & B (95\% Cl) & SE & P value \\
\hline Age (years) & $-16(-119$ to 87$)$ & 52 & 0.765 \\
\hline Gender (male) & $1139(-2628$ to 4906$)$ & 1918 & 0.553 \\
\hline Quality (\%) & $3732(-2457$ to 9920$)$ & 3151 & 0.237 \\
\hline LOS Study-ICU (days) & $-529(-671$ to -387$)$ & 72 & $<0.001$ \\
\hline LOS hospital (days) & $-143(-213$ to -71$)$ & 36 & $<0.001$ \\
\hline Reintubations & $-928(-2.457$ to 602$)$ & 779 & 0.234 \\
\hline Average SOFA & $1608(892$ to 2323$)$ & 364 & $<0.001$ \\
\hline Daily costs $(€)$ & $-7.6(-11$ to -4$)$ & 2 & $<0.001$
\end{tabular}

${ }^{*}$ Quality, adherence to the quality indicator 'early weaning from invasive ventilation'.

ICU, intensive care unit; LOS, length of stay; SOFA, Sequential Organ Failure Assessment. of illness. The fixed variables age, sex and percentage of QI adherence examined did not show significant effects on profitability.

In the linear regression analysis, the LOS on the study-ICU $(p<0.001)$, the LOS in the hospital $(p<0.001)$, the averaged daily SOFA score $(\mathrm{p}<0.001)$ and the averaged daily costs per patient $(\mathrm{p}<0.001)$ were shown to have significant effects on the profitability (table 2). Strong effects were found for the averaged daily SOFA score, which increased profits per case by $€ 1608$ (95\% CI $€ 892$ to $€ 2323)$ for each SOFA point. Furthermore, the LOS on the ICU decreased profits per case for $€ 529$ for every day longer on the ICU. To the best of our knowledge, multivariate regression for economic outcome has not yet been conducted for these factors. The regression model was performed without the admission scores for SAPS II, SOFA and APACHE II. When these scores were included, the statistical significances remained unchanged for the remaining variables that were analysed (see table 2).

Comparing the cumulative parameters of weaning patients along the years (see table 3), a higher number of patients weaned as well as a higher average SOFAscore can be associated with a higher number of median economic result. The observation over time supports the outcome parameters of table 1. Considering the development since 2012, there is an increase in the number of patients weaned per year and a decrease in the median hours of ventilation per patient.

\section{DISCUSSION}

The most important finding was that clinical and economic results were better within the HAG than the LAG. We sought to evaluate whether adherence above a certain quality threshold leads to a better economic result per case for the hospital. Our univariate model confirmed our hypothesis that higher quality leads to better LOS and hospital costs of intensive care patients. However, an improvement of the QI 'early weaning' was not directly associated with a significant impact on the profitability per case. In the regression model, we were not able to prove that more quality lead to higher earnings. Instead, significant factors were clinical outcome parameters (LOS ICU, LOS Hospital and averaged daily SOFA score), which had direct effects on profitability. Moreover, these parameters were also superior within the HAG, indicating a certain quality effect. This sequence of effects shows that quality affects the economic results indirectly via clinical outcome. This means that quality leads to clinical efficiency. Literature already proposes a more effective use of the costly resource ICU. ${ }^{26}$ Thus, from an economic perspective it is recommended to transfer patients as early as possible from ICU downstream (eg, intermediate care) since a prolonged ICU-stay might be inappropriate, dangerous and costly. ${ }^{23} 25$

Highly specialised ICUs are resource-intensive and costintensive and not universally available. By implementing $\mathrm{QM}$ as a method to constantly eliminating the factors 
Table 3 Financial demographics in median over time of 583 patients who underwent the weaning process

\begin{tabular}{|c|c|c|c|c|c|c|}
\hline Variable & 2012 & 2013 & 2014 & 2015 & 2016 & 2017 \\
\hline $\begin{array}{l}\text { Weaning } \\
\text { patients }\end{array}$ & 65 & 82 & 100 & 114 & 125 & 97 \\
\hline $\begin{array}{l}\text { Average } \\
\text { SOFA }\end{array}$ & 7.5 (5.6; 9.3) & $8.3(6.7 ; 11.0)$ & $8.2(6.5 ; 10.1)$ & 8.1 (6.6; 9.6) & $8.9(7.0 ; 10.7)$ & $8.3(6.7 ; 11.0)$ \\
\hline $\begin{array}{l}\text { Duration of } \\
\text { ventilation } \\
\text { (hour) }\end{array}$ & $660(480 ; 977)$ & $451(230 ; 667)$ & $400(206 ; 673)$ & $439(261 ; 720)$ & $374(239 ; 602)$ & $364(210 ; 619)$ \\
\hline $\begin{array}{l}\text { Profits per } \\
\text { case }(€) \dagger\end{array}$ & $-12517(-24$ 848; -806) & -11011 (-28 547; 999) & $-945(-14141 ; 8843)$ & $390(-11340 ; 12201)$ & 3439 (-7494; 8784) & $-3136(-22$ 012; 8284) \\
\hline
\end{tabular}

of chance, hospitals are trying to reduce complexity in defining, measuring and learning from QIs. Furthermore, QM is associated as a necessity for certification processes and therefore incremental part of critical care concepts. ${ }^{1}$ The importance of weaning protocols and according adherence is based on studies that have proven between $70 \%$ and $80 \%$ of all patients receiving $>24$ hour invasive ventilation could already be weaned after the first SBT. $^{82728}$ This is why in 2011, a study at our institution investigated that the support of fast visual feedback for adherence to standard operating procedures within the PDMS led to decreased duration of mechanical ventilation and higher documentation compliance, supporting our findings. ${ }^{29}$ The approach of measuring and steering quality with indicators carries several direct and indirect economic incentives. First, less loss per patient due to better clinical outcome has positive effects on the general economic results of the department. Second, decreased LOS on the ICU gives room to available beds earlier and therefore other patients to fill in the existing resource. ${ }^{30}$ Third, because of public reporting and potential pay for quality structures, indicators are important methods for measuring quality and safety in healthcare, resulting in better outcome. ${ }^{31}$ In particular, transparent QIs allow department leaders to identify weak spots and initiate improvement in a structured and measurable way. ${ }^{2}$ Our matched with a study performed in 2008, showing positive clinical outcome effects of ventilator weaning protocol measures. ${ }^{32}$ Patients spent less time on mechanical ventilation, and thus less time in intensive care and in the hospital. We found that the more patients that could be weaned per year, the less time they spent on the ventilator and better the economic results followed, since more patients generating contribution margins covered fixed costs. This effect shows that redundant capacities can be used for new admissions and thus higher throughput, similar to a former study at our institution. ${ }^{33}$

This study is the first to find that high adherence to the QI 'early weaning from invasive ventilation' above a proven threshold of $65 \%$ showed higher economic returns (or less losses) than low adherence. Furthermore, the study is unique in using a case defined data set to examine the economic effect of a single QI. Current economic prediction models in intensive care usually describe interventions of entire QM programmes ${ }^{30}$ or changes in staffing. ${ }^{33}$ Overall, we found that the median financial return for a hospital is negative when focusing on weaning from ventilation. This is independent of their QI adherence results. In Germany, insurance companies reimburse hospitals using the G-DRG System (German DGRs System) based on a performance-oriented compensation for inpatients. Within DRG-Systems, ${ }^{30}$ the casemix of weaning patients does not provide adequate economic incentives for quality based critical care since the reimbursement is mainly focused on procedures, for example, duration of ventilation. This is consistent with other studies that found higher process quality led to decreased ventilator dependence and reduced reimbursement. ${ }^{25} 2634$ To avoid wrong incentives, reimbursement should potentially be tied to patient-centred outcomes. For example, the prevention of VAP, PICS and CCIs. In this study, we used comprehensive per-patient cost data, based exclusively on the DRG-system. At our institution, a case-related cost calculation is well established and highly accurate for reimbursement per case and costs since we have been substantial cost-accounting reference centre since the beginning of the G-DRG-system. Therefore, we used this administrative data to calculate the economic outcome per case. ${ }^{35}$ In Germany, a representative mix of hospitals gather case-related treatment costs on a yearly basis in order to report them to the Institute for the Hospital Reimbursement System for continuous development. ${ }^{36}$ On an annual basis, cost weights are adjusted for each DRG, potentially leading to higher reimbursement per case. Hospitals can also benefit from economies of scale, considering more cases per year with fixed reimbursement values. This may explain why in 2015 and 2016 profits per case were higher.

The results of this study can inform policy-makers on the following points: In Germany, the application of QIs in critical care is so far not mandatory. ${ }^{12}$ Since positive effects of clinical and economic parameters can be found 
measuring the adherence to only one indicator of the DIVI set $(\mathrm{n}=10)$, it is recommended to establish QIs widely and combine patient-centred outcomes with economic outcomes systematically. Over the years examined, we found that weaning and the according QI have developed positively as the number of patients receiving weaning increased while the duration of ventilation per patient decreased. The relation between these two parameters shows that the quality of care increased and the organisation for the volume effect became more efficient, which is a dominant economic factor according to Nguyen et $a l .{ }^{37}$ However, in order to evolve further in this direction, intensive care needs adequate reimbursement. Higher assessment scores as SAPS II or SOFA play an important role in ICU reimbursement and might induce higher DRG reimbursement. Considering QM, contrary to the majority of ward care, which benefits from shorter LOS within the flat-compensation system, a decrease in LOS in intensive care is not rewarded with higher reimbursement. Literature confirms our analyses. ${ }^{36}$ This is why we recommend that efforts for quality should be shifted in the centre of reimbursement in intensive care for better clinical outcomes, following the approach of valuedbased payment (pay for quality), where ICUs are checked on costs and quality of service. ${ }^{38}$ Furthermore, because keeping patients on the ICU and on mechanical ventilation economically-incentivised is proven to be dangerous for the patient ${ }^{8}$ and inefficient for the organisation. ${ }^{30}$ This structural change can ensure the incentives for intensivists to adhere to quality standards instead of collecting ventilation hours. Our argument is supported by a recent publication of a group of experts in intensive care. They argue in favour for a reform in hospital reimbursement, away from flat-compensation towards progressive levels of intensive care. Moreover, they suggest a central planning of all system relevant intensive care infrastructures and according criteria for quality standards. ${ }^{39}$ In the end, hospitals benefit from investments in quality, as clinical quality has subsequent effects on economic returns. Thus, not only hospitals, insurance companies and policymakers profit from adherence to QIs, also the patient who should be in the centre of healthcare does.

\section{Unanswered questions and future research}

As noted previously, the study was conducted in a tertiary university hospital, which is characterised by specific and well-established medical processes and structures. A transfer of our observations to other ICUs or reimbursement systems is not feasible. The current study is subject to its retrospective design and potential selection bias, as some of the cases with incomplete data or special diagnoses were not detected during the observation period. We could have used neurological and neurosurgical diagnoses to exclude patients with low chances for weaning outcome, but in our administrative system there is no time point matched to it accordingly as diagnoses are often added just before discharge. For example, patients developing specific neurological conditions after their stay on the study-ICU. Some aspects of our analysis deserve comment on limitation. First, the weaning process has constantly evolved during the years between 2012 and 2017. Since the importance of the weaning protocol emerged throughout the years, the focus on measures hereof and according documentation improved over the years as documentation became mandatory at our institution. ${ }^{8}$ Furthermore, it was not possible matching the qualifications of staffing as a determinant of adherence to quality and curbing of costs. There is supposed to be a connection between experience and cost awareness. ${ }^{40}$ Second, even though indicators and our study-ICU can be examined independently for research purposes, the QI and its progression are substantially connected to other intensive care indicators. ${ }^{19}$ For further research, the interactions between the QIs and the progression on other ICUs need to be considered. Our results provide a robust assessment of the impact of changes of the quality adherence and robust evaluation of their effects.

\section{CONCLUSION}

While the need for critical care increases constantly for various reasons (eg, demographic change or pandemic crisis), the challenge to provide high-quality but costeffective services will only become more important. Available resources differ among the various hospital sizes and types. Although we examined a single indicator for quality in a university reference centre and found proof that high adherence to it lead to significantly better clinical outcome, we think patients and hospitals in general benefit from high adherence to quality measures. Within the univariate analysis, major clinical parameters were significantly better in the HAG. Furthermore, we showed that adherence for $65 \%$ or higher generated significantly higher median earnings within our univariate analysis. However, we also showed that the investigated QI does not significantly affect economic results in our multivariate analysis. Instead, by using clinical parameters as proxies for clinical outcome, they were found to be the main drivers for according economic success. The reason for this is the increased number of patients who could be treated due to more total capacity, when LOS decreased due to higher quality. This is why the focus of this study is not only on reimbursement and on costs, but also on the direct effect of quality on the clinical outcome, which subsequently influences economic results.

Overall, quality matters for reimbursement, but reimbursement is not adjusted to the costs of providing quality. Since there is no central, structured and timely publication of comparable quality data in Germany, it is difficult for politics and assurances to reimburse on a pay for quality model as the basis for comparisons is missing as not mandatory. Still, as quality in treatment is decisive for the patient's hospital choice and the results of the treatment, QIs will be essential for public information and health economics as the patient decides where to be treated. 
Contributors CS introduced quality indicator based treatment for critically ill patients at Charité hospital in terms of both research and implementation in patient care. She perceived the underlying idea for this study. CS and FB set the aims and design of this study. They accept full responsibilty for the work, had access to the data and controlled the decision to pubilsh. AZ and RA performed data collection. AZ conducted statistical analysis supervised by JK. AZ shared responsibility for the study design, had full access to the data and drafted the manuscript. CS and OK contributed to the interpretation of data from a medical point of view, and specifically from the perspective of quality indicators. MH and MD contributed from the perspective of economics, RJ from the perspective of quality science. FB supervised the overall coordination of the study and contributed from the data science perspective. All authors critically reviewed and advised with their expertise on the manuscript.

Funding This analysis is part of a quality improvement effort from the Department of Anesthesiology and Operative Intensive Care Medicine of the Charité Universitätsmedizin - Berlin, Campus Mitte and Virchow-Klinikum.

Competing interests CS reports grants from Aridis Pharmaceutical, grants from B. Braun Melsungen AG, grants from Drägerwerk AG \& Co. KGaA, grants from Deutsche Forschungsgemeinschaft/German Research Society, grants from Deutsches Zentrum für Luft- und Raumfahrt e. V. (DLR)/German Aerospace Centre, grants from Einstein Stiftung Berlin/Einstein Foundation Berlin, grants from European Society of Anaesthesiology, grants from Gemeinsamer Bundesausschuss/Federal Joint Committee (G-BA), grants from Inneruniversitäre Forschungsförderung/Inner University Grants, grants from Projektträger im DLR/ Project Management Agency, grants from Stifterverband/Non-Profit Society Promoting Science and Education, grants from WHOCC, grants from Baxter Deutschland, grants from Cytosorbents Europe, grants from Edwards Lifesciences Germany, grants from Fresenius Medical Care, grants from Grünenthal, grants from Masimo Europe, grants from Pfizer Pharma PFE, personal fees from Georg Thieme Verlag, grants from Dr. F. Köhler Chemie, grants from Sintetica, grants from European Commission, grants from Stifterverband für die deutsche Wissenschaft e.V./Philips, grants from Stiftung Charité, grants from AGUETTANT Deutschland, grants from AbbVie Deutschland GmbH \& Co. KG, grants from Amomed Pharma $\mathrm{GmbH}$, grants from InTouch Health, grants from Copra System, grants from Correvio, grants from Max-Planck-Gesellschaft zur Förderung der Wissenschaften e.V., grants from Deutsche Gesellschaft für Anästhesiologie \& Intensivmeidzin (DGAI), grants from Stifterverband für die deutsche Wissenschaft e.V./Medtronic, grants from Philips Electronics Nederland BV, grants from BMH, outside the submitted work; In addition, CS has a patent 102014215211.9 licensed, a patent 102014215212.9 licensed, a patent 102018114364.8 licensed, and a patent 102018110275.5 licensed. MH has nothing to disclose. MD has nothing to disclose. RA has nothing to disclose. JK has nothing to disclose. RJ has nothing to disclose. FB reports grants from Einstein Foundation, personal fees from Axon Publishing, grants from Vifor Pharma, personal fees from Elsevier Publishing, grants from Federal Ministry of Health, Germany, grants from Berlin Institute of Health, outside the submitted work.

Patient consent for publication Not applicable.

Ethics approval The study was approved by the Ethics Commission of Charité Universitätsmedizin Berlin (EA2/139/20). The need for patient's consent was waived due to the retrospective nature of the study.

Provenance and peer review Not commissioned; externally peer reviewed.

Data availability statement Data are available on reasonable request. The datasets analyzed during the current study are available from the corresponding author on reasonable request.

Supplemental material This content has been supplied by the author(s). It has not been vetted by BMJ Publishing Group Limited (BMJ) and may not have been peer-reviewed. Any opinions or recommendations discussed are solely those of the author(s) and are not endorsed by BMJ. BMJ disclaims all liability and responsibility arising from any reliance placed on the content. Where the content includes any translated material, BMJ does not warrant the accuracy and reliability of the translations (including but not limited to local regulations, clinical guidelines, terminology, drug names and drug dosages), and is not responsible for any error and/or omissions arising from translation and adaptation or otherwise.

Open access This is an open access article distributed in accordance with the Creative Commons Attribution Non Commercial (CC BY-NC 4.0) license, which permits others to distribute, remix, adapt, build upon this work non-commercially, and license their derivative works on different terms, provided the original work is properly cited, appropriate credit is given, any changes made indicated, and the use is non-commercial. See: http://creativecommons.org/licenses/by-nc/4.0/.
ORCID iDs

Claudia Spies http://orcid.org/0000-0002-1062-0495

Felix Balzer http://orcid.org/0000-0002-6789-8471

\section{REFERENCES}

1 Holtel M, Roßmüller T, Frommhold K. [Quality Management in Medicine: What the Surgeon Needs to Know]. Zentralb/ Chir 2016;141:583-90.

2 Magunia P, Keller M, Rhode A. [Effects of quality-oriented remuneration]. Unfallchirurg 2016;119:454-6.

3 Busse R, Geissler A, Aaviksoo A, et al. Diagnosis related groups in Europe: moving towards transparency, efficiency, and quality in hospitals? BMJ 2013;346:f3197.

4 Martin J, Neurohr C, Bauer M, et al. [Cost of intensive care in a German hospital: cost-unit accounting based on the InEK matrix] Anaesthesist 2008;57:505-12.

5 Cooper LM, Linde-Zwirble WT. Medicare intensive care unit use: analysis of incidence, cost, and payment. Crit Care Med 2004;32:2247-53.

6 Funk G-C, Anders S, Breyer M-K, et al. Incidence and outcome of weaning from mechanical ventilation according to new categories. Eur Respir J 2010;35:88-94.

7 Cox CE, Carson SS, Govert JA, et al. An economic evaluation of prolonged mechanical ventilation. Crit Care Med 2007;35:1918-27.

8 Deutsche Gesellschaft für Anästhesiologie \& Intensivmedizin. S3Leitlinie invasive Beatmung und Einsatz extrakorporaler Verfahren bei akuter respiratorischer Insuffizienz, Langversion, stand 04.12.2017, 2017. Available: www.intensivmedizin-leipzig.de [Accessed 21 Nov 2018].

9 Ely EW, Baker AM, Dunagan DP, et al. Effect on the duration of mechanical ventilation of identifying patients capable of breathing spontaneously. N Engl J Med 1996;335:1864-9.

10 Pham T, Brochard LJ, Slutsky AS. Mechanical ventilation: state of the art. Mayo Clin Proc 2017;92:1382-400.

11 Cohen IL, Booth FV. Cost containment and mechanical ventilation in the United States. New Horiz 1994;2:283-90.

12 Kumpf O. [Quality indicators in intensive care medicine : Background and practical use]. Med Klin Intensivmed Notfmed 2021;116:17-28.

13 Ihra GC, Lehberger J, Hochrieser H, et al. Development of demographics and outcome of very old critically ill patients admitted to intensive care units. Intensive Care Med 2012;38:620-6.

14 WeanNet Study Group. [WeanNet: The network of weaning units of the DGP (Deutsche Gesellschaft für Pneumologie und Beatmungsmedizin) - results to epidemiology an outcome in patients with prolonged weaning]. Dtsch Med Wochenschr 2016;141:e166-72.

15 Navalesi P, Frigerio P, Patzlaff A, et al. Prolonged weaning: from the intensive care unit to home. Rev Port Pneumol 2014;20:264-72.

16 Braun J-P, Bause H, Bloos F, et al. Peer reviewing critical care: a pragmatic approach to quality management. Ger Med Sci 2010;8:Doc23.

17 Bundesministerium für Gesundheit. Qualitätssicherung im Krankenhausbereich, 2018. Available: https://www.bundesge sundheitsministerium.de/qualitaet-krankenhausversorgung.html [Accessed 3 Sep 2018].

18 Brinkmann A, Braun JP, Riessen R, et al. [Quality assurance concepts in intensive care medicine]. Med Klin Intensivmed Notfmed 2015;110:575-80. 582-583.

19 Kumpf O, Braun J-P, Brinkmann A, et al. Quality indicators in intensive care medicine for Germany - third edition 2017. Ger Med Sci 2017;15:Doc10.

20 Søgaard R, Enemark U. The cost-quality relationship in European hospitals: a systematic review. J Health Serv Res Policy 2017;22:126-33

21 Donabedian A. The role of outcomes in quality assessment and assurance. QRB Qual Rev Bull 1992;18:356-60.

22 Spies C, Kastrup M, Kerner T. SOPs in Anästhesiologie und Schmerztherapie. Thieme 2013.

23 Kastrup M, von Dossow V, Seeling M, et al. Key performance indicators in intensive care medicine. A retrospective matched cohort study. J Int Med Res 2009;37:1267-84.

24 Fadila M, Regunath H. Ventilator weaning. Treasure Island, FL, 2018.

25 Nachtigall I, Tamarkin A, Tafelski S, et al. Impact of adherence to standard operating procedures for pneumonia on outcome of intensive care unit patients. Crit Care Med 2009;37:159-66.

26 Rapoport J, Teres D, Zhao Y, et al. Length of stay data as a guide to hospital economic performance for ICU patients. Med Care 2003;41:386-97. 
27 Brochard L, Rauss A, Benito S, et al. Comparison of three methods of gradual withdrawal from ventilatory support during weaning from mechanical ventilation. Am J Respir Crit Care Med 1994;150:896-903.

28 Vitacca M, Vianello A, Colombo D, et al. Comparison of two methods for weaning patients with chronic obstructive pulmonary disease requiring mechanical ventilation for more than 15 days. Am J Respir Crit Care Med 2001;164:225-30.

29 Kastrup M, Nolting MJ, Ahlborn R, et al. An electronic tool for visual feedback to monitor the adherence to quality indicators in intensive care medicine. J Int Med Res 2011;39:2187-200.

30 Smyrnios NA, Connolly A, Wilson MM, et al. Effects of a multifaceted, multidisciplinary, hospital-wide quality improvement program on weaning from mechanical ventilation. Crit Care Med 2002;30:1224-30.

31 Lindenauer PK, Remus D, Roman S, et al. Public reporting and pay for performance in hospital quality improvement. $N$ Engl J Med 2007:356:486-96.

32 Girard TD, Kress JP, Fuchs BD, et al. Efficacy and safety of a paired sedation and ventilator weaning protocol for mechanically ventilated patients in intensive care (awakening and breathing controlled trial): a randomised controlled trial. Lancet 2008;371:126-34.
33 Kastrup M, Seeling M, Barthel S, et al. Effects of intensivist coverage in a post-anaesthesia care unit on surgical patients' case mix and characteristics of the intensive care unit. Crit Care 2012;16:R126.

34 Soo Hoo GW, Wen YE, Nguyen TV, et al. Impact of clinical guidelines in the management of severe hospital-acquired pneumonia. Chest 2005;128:2778-87.

$35 \mathrm{InEK} \mathrm{GmbH}$. Kalkulation von Behandlungskosten, 2016. Available: https://www.g-drg.de/Kalkulation2/DRG-Fallpauschalen_17b_KHG/ Kalkulationshandbuch

36 Riessen R, Hermes C, Bodmann K-F, et al. [Reimbursement of intensive care services in the German DRG system : Current problems and possible solutions]. Med Klin Intensivmed Notfmed 2018;113:13-23.

37 Nguyen Y-L, Wallace DJ, Yordanov Y, et al. The volume-outcome relationship in critical care: a systematic review and meta-analysis. Chest 2015;148:79-92.

38 Blumenthal D, Abrams MK. Tailoring complex care management for High-Need, high-cost patients. JAMA 2016;316:1657-8.

39 Riessen R, Markewitz A, Grigoleit M, et al. [Discussion paper for a hospital financing reform in Germany from the perspective of intensive care medicine]. Med Klin Intensivmed Notfmed 2020;115:59-66

40 Wunsch H, Gershengorn H, Scales DC. Economics of ICU organization and management. Crit Care Clin 2012;28:25-37. v. 